\title{
Effect of pioglitazone, quercetin and hydroxy citric acid on inflammatory markers in experimentally induced non alcoholic steatohepatitis (NASH).
}

\author{
Surapaneni Krishna Mohan*1, Mallika Jainu² \\ *1Department of Biochemistry, Saveetha Medical College and Hospital, Faculty of Medicine, Saveetha University, Saveetha Nagar, \\ Thandalam, Chennai - 602 105, Tamilnadu, India. \\ ${ }^{2}$ Department of Biomedical Engineering, SSN Engineering College, OMR, Klavakkam, Chennai - 603 110, Tamilnadu, India.
}

\begin{abstract}
Background: Non Alcoholic Fatty Liver Disease (NAFLD) spectrum comprises of diseases ranging from simple steatosis to steatohepatitis. Non-Alcoholic Steatohepatitis (NASH), an asymptomatic disease, characterized by the fatty infiltration with inflammation is a key component of NAFLD spectrum that may proceed to the liver cirrhosis, the end stage liver disease, if not diagnosed and treated properly. Inflammatory mediators have been investigated as potential diagnostic tools to understand the pathogenesis of NASH. In this study, the comparative effect of pioglitazone, quercetin and hydroxy citric acid on the levels of inflammatory markers in experimentally induced NASH has been studied. Methods: The experimental protocol consists of 48 Male Wister rats, which were divided into 8 groups. The levels of tumor necrosis factor- $\alpha$ (TNF- $\alpha$ ) and myeloperoxidase (MPO) were estimated in experimental NASH. Results: A significant increase in the levels of inflammatory markers such as tumor necrosis factor- $\alpha$ (TNF- $\alpha$ ) and myeloperoxidase (MPO) was noticed in experimental NASH rats as compared to control group. On treatment with pioglitazone and quercetin, they showed significant decrease in the levels of TNF- $\alpha$ and MPO. Whereas, on treatment with hydroxy citric acid, no significant effect on the levels of inflammatory markers viz. TNF- $\alpha$ and MPO were observed. Conclusion: By virtue of our findings, it could be inferred that Quercetin and pioglitazone offers protection against NASH by ameliorating the inflammation (hepatitis), a principle and key feature of $\mathrm{NASH}$, whereas hydroxy citric acid offers very little protection against NASH.
\end{abstract}

Key words: Pioglitazone, Quercetin, Hydroxy citric acid, TNF- $\alpha$, myeloperoxidase, NonAlcoholic Fatty Liver Disease (NAFLD), Non-Alcoholic Steatohepatitis (NASH).

\section{INTRODUCTION}

Non Alcoholic Fatty Liver Disease (NAFLD) represents a spectrum of disease ranging from hepatocellular steatosis through steatohepatitis to fibrosis and irreversible cirrhosis. The prevalence of NAFLD has risen rapidly in parallel with the dramatic rise in obesity and diabetes. ${ }^{1,2}$ Non-Alcoholic Steatohepatitis (NASH) is a form of metabolic liver disease in which, steatosis is associated with lobular inflammation, hepatocyte injury and/or hepatic fibrosis. ${ }^{3,4}$ NASH typically causes no symptoms. When present, clinical features such as fatigue, hepa- tomegaly and aching hepatic discomfort and most of them are non-specific. ${ }^{5}$ In $20-25 \%$ of cases, NASH may progress to advanced stages of hepatic fibrosis, cirrhosis and liver failure then becomes the most common cause of death. ${ }^{6-10}$ Occasionally, hepatocellular carcinoma (HCC) may also occur. The mechanism of progression from the steatosis of NAFLD to the necro-inflammatory state of NASH is poorly understood. However, inflammatory mediators have also been investigated as potential diagnostic tools.
Submission Date : 27-11-2014 Revision Date : :23-12-2014 Accepted Date : :07-01-2015

DOI: $10.5530 /$ ijper.49.2.12 Correspondence Address Dr. Surapaneni Krishna Mohan

Associate Professor \& Vice Principal, Department of Biochemistry Saveetha Medical College and Hospital,

Faculty of Medicine, Saveetha University, Saveetha Nagar, Thandalam

Chennai-602105,

Tamilnadu, India.

E-mail:krishnamohan.surapaneni@gmail.com

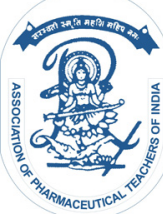

www.ijper.org 
It is important to study the tumor necrosis factor alpha (TNF-a) and Myeloperoxidase (MPO) levels in NASH, since the imbalance of these important inflammatory markers may play an important role in the development of NASH. TNF- $\alpha$, a cytokine is a pro-inflammatory mediator and plays a distinct role in hepatocyte inflammation and cell death. ${ }^{11}$ Also, it is one of the major proinflammatory cytokines involved in the pathogenesis of chronic inflammatory diseases and is modulated by oxidative stress. ${ }^{12} \mathrm{TNF}-\alpha$ also triggers the cellular release of other cytokines, chemokines, or inflammatory mediators and displays antiviral and antimicrobial effects. MPO possesses potent antimicrobial activity and is an indicator of neutrophilic degranulation and was reported to play a crucial role in tissue injury. ${ }^{13,14}$

In our previous studies we have reported the effect of pioglitazone, quercetin, and hydroxy citric acid (HCA) on the hepatic bio markers, lipid profile and lipoproteins, cytochrome P450 2E1 (CYP2E1) in experimentally induced non-alcoholic steatohepatitis (NASH). ${ }^{15-17}$

We have studied the comparative effect of pioglitazone, quercetin and hydroxy citric acid on the status of lipid peroxidation and antioxidants in experimental non-alcoholic steatohepatitis. ${ }^{18}$ This study explores the comparative effects of pioglitazone, quercetin, and hydroxy citric acid on the levels of TNF- $\alpha$ and MPO, the major inflammatory markers in experimentally induced NASH.

\section{MATERIALS AND METHODS}

The experimental model of NASH in rats was established by feeding the animals a high-fat diet for eight weeks, ${ }^{18,19}$ and this model was used to conduct a comparative study of the role of pioglitazone, quercetin, and hydroxy citric acid on various inflammatory marker parameters in non-alcoholic steatohepatitis. Male Wistar rats weighing approximately $250 \mathrm{~g}$ were housed in solid-bottomed polypropylene cages under strict veterinary supervision and maintained in control rooms with a 12-h light/12-h dark cycle. The animals received water and a commercial rat diet, standard diet, or high-fat diet ad libitum according to the experimental protocol. This study conformed to the guiding principles of the Institutional Animal Ethical Committee (IAEC), the Committee for the Purpose of the Control and Supervision of Experiments on Animals (CPCSEA), and the Guide for the Care and Use of Laboratory Animals (IAEC Approval Numbers: 001/006/2010 and 01/007/2011).

The male Wistar rats selected for the study were divided into eight groups as follows: ${ }^{18-20}$

Group 1, Controls $(n=6)$ : The control rats received the regular standard diet for eight weeks.
Group 2, NASH $(n=6)$ : The rats were fed a high-fat diet for eight weeks to induce NASH.

Group 3, pioglitazone control $(n=6)$ : These rats were fed the standard diet for four weeks and were then fed the standard diet and intragastrically administered pioglitazone (4 mg/kg. b.wt.; 0.5\% methyl cellulose w/v) for the next four weeks.

Group 4, quercetin control $(n=6)$ : These rats were fed the standard diet for four weeks and were then fed the standard diet intragastrically administered quercetin $(20 \mathrm{mg} / \mathrm{kg}$. b.wt.) dissolved in 1\% DMSO v/v for the next four weeks.

Group 5, hydroxy citric acid Control $(n=6)$ : These rats were fed the standard diet for four weeks and were then fed the standard diet and intragastrically administered hydroxy citric acid (150 mg/kg. b.wt.) for the next four weeks.

Group 6, NASH + pioglitazone $(n=6)$ : These rats were fed a high-fat diet for four weeks and were then fed the high-fat diet and intragastrically administered pioglitazone (4 mg/kg. b.wt.; 0.5\% methyl cellulose w/v) for the next four weeks.

Group 7, NASH + quercetin $(n=6)$ : These rats were fed a high-fat diet for four weeks and were then fed the high-fat diet and intragastrically administered quercetin (20 mg/kg. b.wt.) dissolved in $1 \% \mathrm{DMSO} \mathrm{v} / \mathrm{v}$ for the next four weeks.

Group 8, NASH + hydroxy citric acid $(\mathrm{n}=6)$ : These rats were fed a high-fat diet for four weeks and were then fed the high-fat diet and intragastrically administered hydroxy citric acid (150 mg/kg. b.wt.) for the next 4 weeks.

After the experimental period, the animals were sacrificed after $12 \mathrm{~h}$ of fasting by cervical decapitation. The blood was collected and centrifuged for $5 \mathrm{~min}$ at 3000 $\mathrm{rpm} / \mathrm{min}$, and the serum was stored at $-70^{\circ} \mathrm{C}$ until various biochemical analysis were conducted.

The levels of tumor necrosis factor $-\alpha(\mathrm{TNF}-\alpha)$ was assayed by the method as described by Konturek et $\mathrm{al}^{21}$ as described by Konturek et al and The myeloperoxidase (MPO) activity was assayed by the method as described by Krawisz et al. ${ }^{22}$

\section{RESULTS}

Histopathological studies after the ingestion of the high-fat diet for 8 weeks revealed all the prominent characteristic features of NASH including steatosis, inflammation, which mimics the NASH in humans. ${ }^{18}$ The treatment with the selected drugs of choice alone doesn't cause any deleterious effects and did not alter the normal metabolism. Inflammation with no fatty degeneration was observed on treatment with pioglitazone $^{18}$ and local hepatocyte necrosis with inflammatory collections was seen on treatment with hydroxy citric 
Table 1: Effect of Pioglitazone, Quercetin and Hydroxy Citric Acid on the Levels of Inflammatory Markers in

\begin{tabular}{|l|c|c|c|c|c|c|c|c|}
\hline Parameter & $\begin{array}{c}\text { Group 1: } \\
\text { Control }\end{array}$ & $\begin{array}{c}\text { Group 2: } \\
\text { NASH }\end{array}$ & $\begin{array}{c}\text { Group 3: } \\
\text { Pioglitazone } \\
\text { Control }\end{array}$ & $\begin{array}{c}\text { Group 4: } \\
\text { Quercetin } \\
\text { Control }\end{array}$ & $\begin{array}{c}\text { Group 5: } \\
\text { HCA } \\
\text { Control }\end{array}$ & $\begin{array}{c}\text { Group 6: } \\
\text { NASH+ } \\
\text { Pioglitazone }\end{array}$ & $\begin{array}{c}\text { Group 7: } \\
\text { NASH+ } \\
\text { Quercetin }\end{array}$ & $\begin{array}{c}\text { Group 8: } \\
\text { NASH + } \\
\text { HCA }\end{array}$ \\
\hline $\begin{array}{l}\text { TNF-alpha } \\
\text { (ng/ml) }\end{array}$ & $8.75 \pm 0.51$ & $12.14 \pm 1.0^{*}$ & $8.83 \pm 0.71$ & $8.51 \pm 0.69$ & $9.01 \pm 0.82$ & $9.14 \pm 0.8^{\circ}$ & $8.02 \pm 0.5^{\mathrm{b}}$ & $9.64 \pm 0.7$ \\
\hline $\begin{array}{l}\text { Plasma MPO } \\
\text { (ng/ml) }\end{array}$ & $40.6 \pm 3.8$ & $59.3 \pm 4.3^{*}$ & $39.2 \pm 2.6$ & $41.5 \pm 3.8$ & $43.6 \pm 4.1$ & $46.9 \pm 3.5^{\circ}$ & $44.3 \pm 3.2^{\mathrm{b}}$ & $53.2 \pm 4.9$ \\
\hline $\begin{array}{l}\text { Values are mean } \pm \text { SEM for } 6 \text { animals in each group. *P<0.001 compared to control group; }{ }^{\circ} \mathrm{P}<0.001 \text { compared to NASH group; } \mathrm{bP}<0.01 \text { compared to NASH group; } \\
\text { P }<0.05 \text { compared to NASH group }\end{array}$ \\
\hline
\end{tabular}

acid. ${ }^{18}$ But, hepatocytes appear mere normal with no obvious fatty and inflammatory changes on treatment with quercetin. ${ }^{18}$

Inflammatory mediators have also been investigated as potential diagnostic tools. NASH was associated with an increase in tumor necrosis factor alpha $(\mathrm{TNF}-\alpha)$ and MPO levels and the imbalance may play an important role in the development of NASH. The pattern of inflammatory markers has been depicted in the Table- 1 and Figure 1-2. A significant increase in the levels of inflammatory markers such as tumor necrosis factor- $\alpha$ (TNF- $\alpha$ ) and myeloperoxidase (MPO) was noticed in experimental NASH rats (group 2) as compared to control group (group 1). The experimental NASH rats treated with pioglitazone (group 6; NASH+pioglitazone) and rats treated with quercetin (group 7; NASH+quercetin) showed significant decrease in the levels of TNF- $\alpha$ and MPO. Whereas, the experimental NASH rats treated with hydroxy citric acid (group 8; NASH+HCA) does not show any significant effect on the levels of inflammatory markers viz. TNF- $\alpha$ and MPO. Rats fed with

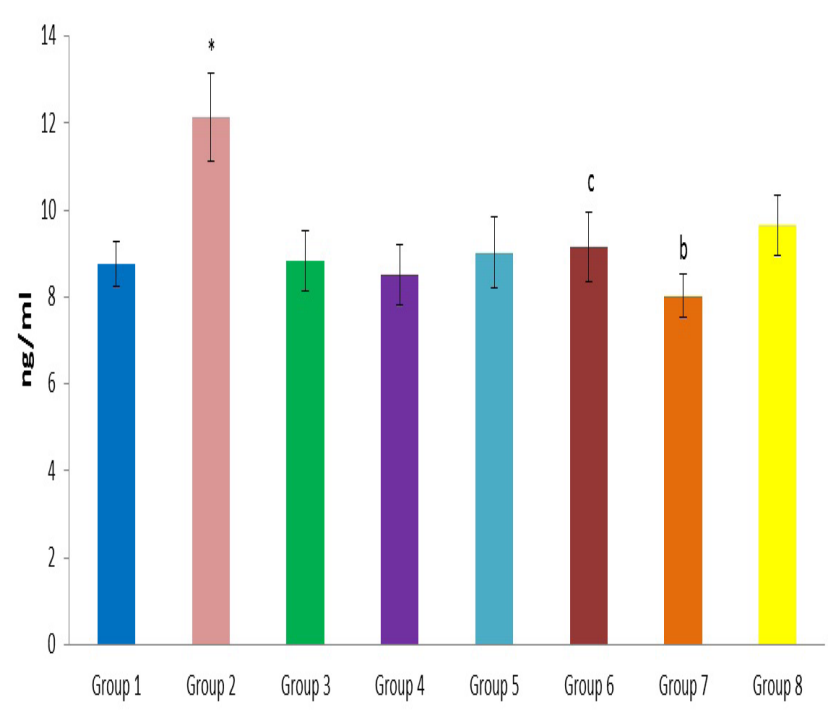

Figure 1: Effect of Pioglitazone, Quercetin and Hydroxy Citric Acid on the Levels of Tumor Necrosis Factor-Alpha (Tnf- $\alpha$ ) In Experimental NASH.

* $\mathrm{P}<0.001$ compared to control group; ${ }^{\mathrm{a} P} \mathrm{P}<0.001$ compared to NASH group; ${ }^{\mathrm{b} P}<0.01$ compared to NASH group; ${ }^{\mathrm{C} P}<0.05$ compared to NASH group standard diet simultaneously with pioglitazone (group 3; pioglitazone control), with quercetin (group 4; quercetin control) and with hydroxy citric acid (group 5; HCA control) does not show any significant effect on the inflammatory markers compared to control group (group 1).

\section{DISCUSSION}

A significant increase in the levels of inflammatory markers such as tumor necrosis factor- $\alpha(\mathrm{TNF}-\alpha)$ and myeloperoxidase (MPO) was noticed in experimental NASH rats (group 2) as compared to control group (group 1). The enhanced levels of TNF- $\alpha$ in NASH in turn activates specific redox sensitive kinases. ${ }^{23}$ These activated specific redox sensitive kinases up regulate the pro-inflammatory pathways resulting in the enhanced insulin resistance which is believed to play a key role in the pathogenesis of NASH. ${ }^{23}$

Cytokine production is increased in nonalcoholic steatohepatitis and is believed to play a role in its pathogenesis. In the liver, tumor necrosis factor-alpha can contribute to oxidative stress ${ }^{24,25}$ and may contribute

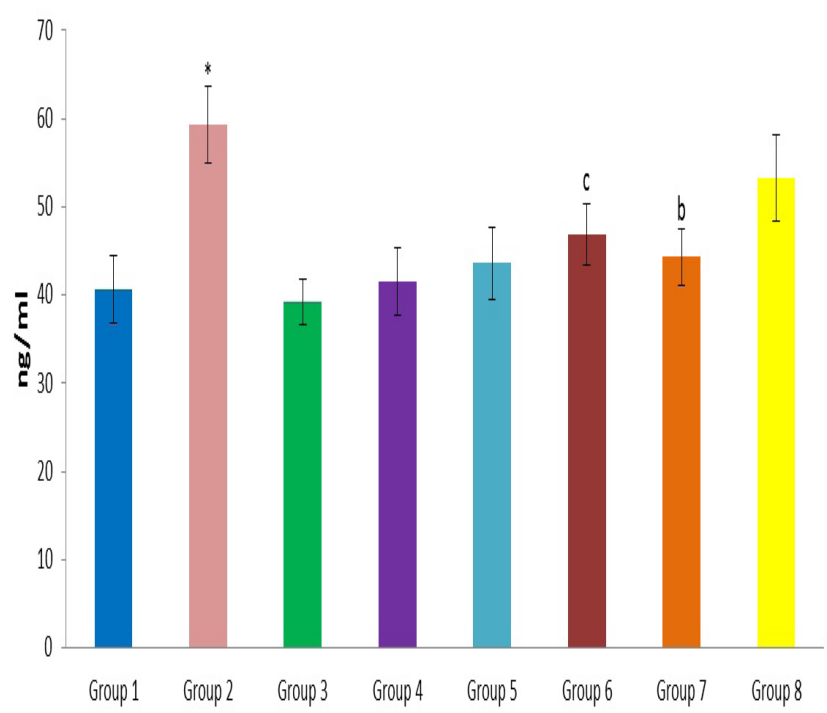

Figure 2: Effect of Pioglitazone, Quercetin and Hydroxy Citric Acid on the Levels of Plasma Myeloperoxidase (MPO) In Experimental NASH.

*P<0.001 compared to control group; ${ }^{\text {a }} \mathrm{P}<0.001$ compared to NASH group; ${ }^{\text {b }} \mathrm{P}<0.01$ compared to $\mathrm{NASH}$ group; ${ }^{\mathrm{C}} \mathrm{P}<0.05$ compared to $\mathrm{NASH}$ group 
to insulin resistance through activation of the inhibitor of kappa kinase beta. ${ }^{23}$ It is suggested that the cytokine TNF- $\alpha$ can be inhibited by quercetin, which may be of clinical significance in host defense mechanisms against various infections. ${ }^{26}$

Myeloperoxidase (MPO) is an important neutrophil enzyme that can generate aggressive oxidants. ${ }^{27}$ One of the principal molecules released after recruitment and activation of phagocytes is myeloperoxidase (MPO), an important enzyme involved in the generation of reactive oxygen species. ${ }^{13}$

Inflammation and oxidative stress are considered critical factors in the progression of nonalcoholic fatty liver disease. ${ }^{17,18,28}$ In the presence of physiological chloride concentrations, MPO reacts with hydrogen peroxide $\left(\mathrm{H}_{2} \mathrm{O}_{2}\right.$, formed by the respiratory burst) to catalyze formation of hypochlorous acid/ hypochlorite and other oxidizing species. ${ }^{13,27}$ These oxidants may contribute to host tissue damage at sites of inflammation through reactions with a wide range of biological substrates, including DNA, lipids, and protein amino groups. ${ }^{29}$ In the absence of physiological chloride concentrations, the $\mathrm{MPO}-\mathrm{H}_{2} \mathrm{O}_{2}$ system can also form reactive nitrogen species ${ }^{30}$ that may initiate lipid peroxidation. Interestingly, macrophages are known to generate high amounts of reactive oxygen and nitrogen species. Therefore, MPO-containing macrophages/ Kupffer cells could play a role in NASH. ${ }^{31}$ MPO activity might be a driving factor underlying the progression of human NASH. ${ }^{32,33}$

To analyze the effect of pioglitazone, quercetin and hydroxy citric acid alone on the liver and on normal metabolic activities, we have chosen to have three drug control groups. Rats fed with standard diet simultaneously with pioglitazone (group 3; pioglitazone control), with quercetin (group 4; quercetin control) and with hydroxy citric acid (group 5; HCA control) does not show any significant effect on the inflammatory markers compared to control group (group 1). All the three drugs did not produce any significant alterations in the levels of inflammatory markers as evidenced by the Table-1 and Figure 1-2.

The experimental NASH rats treated with pioglitazone (group 6; NASH+pioglitazone) and rats treated with quercetin (group 7; NASH+quercetin) showed significant decrease in the levels of TNF- $\alpha$ and MPO. Pioglitazone stimulate maturation of visceral fat, and hence change the adipocytokine profile secreted by adipose tissue. Pioglitazone leads to an increase in adiponectin levels, which counteracts pro-inflammatory cytokines such as tumor necrosis factor- $\alpha$ (TNF $\alpha$ ) and promotes beta oxidation of fatty acids via adenosine monophosphate-activated protein kinase (AMP-K) activation. ${ }^{34,35}$ The increase in beta oxidation, immature adipose tissue in conjunction with a reduction in de novo lipogenesis, decreases gluconeogenesis. ${ }^{36,37}$ Quercetin is an effective inhibitor of human myeloperoxidase (MPO) activity, both with purified enzyme and in a system using stimulated human neutrophils. Moreover, quercetin is directly able to scavenge hypochlorous acid $(\mathrm{HOCl})$, a chlorinated species generated by the, $\mathrm{MPO} / \mathrm{H} 2 \mathrm{O} 2 / \mathrm{Cl}-$ system. $^{38}$

In this present study, quercetin showed protective effect against NASH by significant reduction in inflammatory markers due to its anti-inflammatory activity. Quercetin inhibits TNF- $\alpha$ and IL- 6 , simultaneously induces IL-10 release, and thus evokes the anti-inflammatory effect. ${ }^{39}$ Quercetin also inhibits STAT-1 and NF- $x \mathrm{~B}$ and thus possesses the anti-inflammatory effect. ${ }^{40}$ Quercetin's anti-inflammatory activity may also have a beneficial effect on the muscle damage experienced by athletes after intense exercise. ${ }^{41}$ It has been demonstrated that quercetin can inhibit nuclear factor-kappa $\mathrm{B}(\mathrm{NF}-\boldsymbol{x B}),{ }^{41}$ a chemical in the body which has been shown to play a central role in regulating the immune response to inflammation. Cell culture studies and in vivo (animal) studies have provided evidence supporting quercetin's anti-inflammatory effects. ${ }^{41}$

Whereas, the experimental NASH rats treated with hydroxy citric acid (group 8; NASH+HCA) does not show any significant effect on the levels of inflammatory markers viz. TNF- $\alpha$ and MPO. Hydroxy citric acid which is a phytoconstituent present in the Garcinia Indica or in general Garcinia species, and is a proven anti obesity agent with the lipid lowering actions. ${ }^{42-44}$ It's been well established as a lipid lowering agent and Hypocholesterolemic agent. ${ }^{42-44}$ Recent research showed that it also possesses little anti-inflammatory activity, apart from these two properties ${ }^{45}$. But, in the present study the experimental NASH rats treated with hydroxy citric acid (group 8; NASH+HCA) does not show any significant effect on the levels of inflammatory markers, showing that HCA does not possess any anti-inflammatory action. Due to this, HCA can confer very less protection against NASH, since inflammation of the liver (hepatitis) is one of the principle and key features of NASH.

\section{CONCLUSION}

TNF- $\alpha$ and MPO have long been recognized for their pro-inflammatory properties and their role in NASH progression was clearly established. TNF- $\alpha$ and MPO were highly expressed in experimentally induced NASH group, compared to control group. Quercetin and pioglitazone offers protection against NASH by ameliorating the inflammation (hepatitis), a principle and key feature of NASH, whereas hydroxy citric acid offers very little protection against $\mathrm{NASH}$. 


\section{CONFLICTS OF INTEREST}

Authors declared no conflicts of interest

\section{ACKNOWLEDGEMENTS}

The Authors are very much thankful to Dr.S.Porchelvan, M.Sc., M.B.A., PGDCA.,PhD., Professor in Biostatistics, Saveetha Medical College \& Hospital, Saveetha University for assisting us in performing the statistical analyses.

\section{REFERENCES}

1. Charlton M. Nonalcoholic fatty liver disease: a review of current understanding and future impact. Clin Gastroenterol Hepatol. 2004; 2(12): 1048-58.

2. Vuppalanchi R, Chalasani N. Nonalcoholic fatty liver disease and nonalcoholic steatohepatitis: Selected practical issues in their evaluation and management. Hepatology 2009; 49(1): 306-17.

3. Ludwig J, Viggiano TR, McGill DB, Oh BJ. Nonalcoholic steatohepatitis: Mayo Clinic experiences with a hitherto unnamed disease. Mayo Clin Proc. 1980; 55(7): 434-8.

4. Ahmed MH, Byrne CD. Non-alcoholic steatohepatitis. In Byrne CD, D Wild S, editors. Metabolic syndrome. Chichester: John Wiley \& Sons; 2005. pp. 279-305.

5. Liou I, Kowdley KV. Natural history of nonalcoholic steatohepatitis. J Clin Gastroenterology 2006; 40(Suppl 1): S11-6.

6. Bugianesi E, Leone N, Vanni E, Marchesini G, Brunello F, Carucci P, et al. Expanding the natural history of nonalcoholic steatohepatitis: from cryptogenic cirrhosis to hepatocellular carcinoma. Gastroenterology 2002; 123(1): 134-40.

7. McCullough AJ. The epidemiology and risk factors of NASH. In Farrell GC, George J, de la M Hall P, McCullough AJ, editors. Fatty liver disease: NASH and related disorders. Oxford: Blackwell; 2005. pp. 23-37.

8. Heidelbaugh JJ, Bruderly M. Cirrhosis and chronic liver failure: Part I. Diagnosis and evaluation. Am Fam Phys. 2006; 74(5): 756-62.

9. Farrell GC, Larter CZ. Nonalcoholic fatty liver disease: from steatosis to cirrhosis. Hepatology 2006; 43(Suppl 1): S99-112.

10. Pascale A, Pais R, Ratziu V. An overview of nonalcoholic steatohepatitis: past, present and future directions. J Gastrointestin Liver Dis. 2010; 19(4): 415.

11. Hui JM, Hodge A, Farrell GC, Kench JG, Kriketos A, George J. Beyond insulin resistance in NASH: TNF-alpha or adiponectin? Hepatology 2004; 40(1): 46-54.

12. Aggarwal BB. Tumour necrosis factor receptor associated signaling molecules and their role in activation of apoptosis. JNK and NF-kappa B. Ann. Rheum. Dis. 2000; 59(S1): 6-16.

13. Klebanoff SJ. Myeloperoxidase: friend and foe. J Leukoc Biol. 2005; 77(5): 598-625.

14. Krawisz JE, Sharon P, Stenson WF. Quantitative assay for acute intestinal inflammation based on myeloperoxidase activity. Assessment of inflammation in rat and hamster models. Gastroenterology 1984; 87(6): 1344-50.

15. Surapaneni KM, Jainu M. Pioglitazone, quercetin and hydroxy citric acid effect on hepatic biomarkers in Non Alcoholic Steatohepatitis. Pharmacognosy Res. 2014; 6(2): 153-62.

16. Surapaneni KM, Jainu M. Effect of pioglitazone, quercetin, and hydroxy citric acid on the lipid profile and lipoproteins in experimentally induced nonalcoholicsteatohepatitis (NASH). Indian J Pharm Educ Res. 2014; 48(1) 32-8.

17. Surapaneni KM, Priya VV, Mallika J. Pioglitazone, quercetin and hydroxy citric acid effect on cytochrome P450 2E1 (CYP2E1) enzyme levels in experimentally induced non alcoholic steatohepatitis (NASH). Eur Rev Med Pharmacol Sci. 2014; 18(18): 2736-41.

18. Surapaneni KM, Jainu M. Comparative effect of pioglitazone, quercetin and hydroxy citric acid on the status of lipid peroxidation and antioxidants in experimental non-alcoholic steatohepatitis. J Phyiol Pharmacol. 2014; 65(1): 67-74.

19. Surapaneni KM, Saraswathi P, Jainu M. Non alcoholic steatohepatitis (NASH) experimental model induction in rats. Int J Pharm Bio Sci. 2012 July; 3(3): (B) 1085- 90.
20. Surapaneni KM, Sarswathi P, Jainu M. Role of pioglitazone, quercetin and hydroxy citric acid against non alcoholic steatohepatitis (NASH) - histological and scanning electron microscopy (SEM) studies in an experimental model of NASH. Asian J Pharm Clin Res. 2012; 5(4): 244-7.

21. Konturek PC, Brozozowski T, Konturek SJ, Kwiecien S, Dembinski A, Hahn EG. Influence of bacterial lipopolysaccharide on healing of chronic experimental ulcer in rat. Scand J Gastroenterol 2001; 36(12): 1239-47.

22. Krawisz JE, Sharon P, Stenson WF. Quantitative assay for acute intestinal inflammation based on myeloperoxidase activity. Assessment of inflammation in rat and hamster models. Gastroenterology 1984; 87(6): 1344-50.

23. Yuan $\mathrm{M}$, Konstantopoulos $\mathrm{N}$, Lee $\mathrm{J}$, et al. Reversal of obesity and diet indenced insulin resistance with salicylates or targeted distruption of IKKbeta. Science 2001; 293 (5535): 1673-7.

24. Tilg $\mathrm{H}$, Diehl AM. Cytokines in alcoholic and nonalcoholic steatohepatitis. $\mathrm{N}$ Engl J Med. 2000; 343(20): 1467-76.

25. Tilg H, Hotamisligil GS. Nonalcoholic fatty liver disease: Cytokine- adipokine interplay and regulation of insulin resistance. Gastroenterology 2006; 131(3): 934-45.

26. Madhavan Nair P, Supriya Mahajan, Jessica Reynolds L, Ravikumar Aalinkeel, Harikrishnan Nair, et al. The Flavonoid Quercetin Inhibits Proinflammatory Cytokine (Tumor Necrosis Factor Alpha) Gene Expression in Normal Peripheral Blood Mononuclear Cells via Modulation of the NF-к $\beta$ System. Clin Vaccine Immunol. 2006 March; 13(3): 319-28.

27. Nauseef WM, et al. Biosynthesis and processing of myeloperoxidase-a marker for myeloid cell differentiation. Eur J Haematol. 1998; 40(2): 97-110.

28. Schults MA, Nagle PW, Rensen SS, Godschalk RW, Munnia A, Peluso M, et al. Decreased nucleotide excision repair in steatotic livers associates with myeloperoxidase-immunoreactivity. Mutat Res. Aug1, 2012; 736(1-2): 75-81. Epub 2011 Nov 7.

29. Davies MJ, Hawkins CL, Pattison DI, Rees MD. Mammalian heme peroxidases: from molecular mechanisms to health implications. Antioxid Redox Signal 2008; 10(7): 1199-234.

30. Hazen SL, Zhang R, Shen Z, Wu W, Podrez EA, MacPherson JC, et al. Formation of nitric oxide-derived oxidants by myeloperoxidase in monocytes: pathways for monocyte-mediated protein nitration and lipid peroxidation in vivo. Circ Res. 1999; 85(10): 950-958.

31. Mantovani A, Sica A, Locati M. New vistas on macrophage differentiation and activation. Eur J Immunol. 2007; 37(1): 14-6.

32. Malle E, Furtmuller PG, Sattler W, Obinger C. Myeloperoxidase: a target for new drug development? Br J Pharmacol. 2007; 152(6): 838-54.

33. Sander Rensen S, Yanti Slaats, Jeroen Nijhuis, Anneke Jans, Veerle Bieghs, Ann Driessen, et al. Increased Hepatic Myeloperoxidase Activity in Obese Subjects with Nonalcoholic Steatohepatitis. Am J Pathol. October 2009; 175(4): 1473-82.

34. Coletta DK, Sriwijitkamol A, Wajcberg E, Tantiwong P, Li M, Prentki M, et al. Pioglitazone stimulates AMP-activated protein kinase signalling and increases the expression of genes involved in adiponectin signalling, mitochondrial function and fat oxidation in human skeletal muscle in vivo: a randomised trial. Diabetologia 2009 Apr; 52(4): 723-32. Epub 2009 Jan 24.

35. Miyazaki Y, Mahankali A, Wajcberg E, Bajaj M, Mandarino LJ, DeFronzo RA. Effect of pioglitazone on circulating adipocytokine levels and insulin sensitivity in type 2 diabetic patients. J Clin Endocrinol Metab. 2004; 89(9): 4312-9.

36. Lutchman G, Modi A, Kleiner DE, Promrat K, Heller T, Ghany M, et al. The effects of discontinuing pioglitazone in patients with nonalcoholic steatohepatitis. Hepatology 2007; 46(2): 424-9.

37. Miyazaki Y, Mahankali A, Wajcberg E, Bajaj M, Mandarino LJ, DeFronzo RA. Effect of pioglitazone on circulating adipocytokine levels and insulin sensitivity in type 2 diabetic patients. J Clin Endocrinol Metab. 2004; 89(9): 4312-9.

38. Pincemail J, Deby C, Thirion A, de Bruyn-Dister M, Goutie R. Human myeloperoxidase activity is inhibited in vitro by quercetin. Comparison with three related compounds. Cellular and Molecular Life Sciences 1988; 44(5): 450-3.

39. Comalada M, Ballester I, Bailón E, Sierra S, Xaus J, Gálvez J, et al. Inhibition of pro-inflammatory markers in primary bone marrow derived mouse macrophages by naturally occurring flavonoids: analysis of the structureactivity relationship. Biochem Pharmacol. 2006; 72(8): 1010-21.

40. Mari Ham“ al" ainen, Riina Nieminen, Pia Vuorela, Marina Heinonen, Eeva Moilanen. Anti-Inflammatory Effects of Flavonoids: Genistein, Kaempferol, Quercetin, and Daidzein Inhibit STAT-1 and NF-KB Activations, Whereas 
Flavone, Isorhamnetin, Naringenin, and Pelargonidin Inhibit only NF-KB Activation along with Their Inhibitory Effect on iNOS Expression and NO Production in Activated Macrophages. Mediators of Inflammation. 2007; 2007: Article ID 45673 1-10.

41. Harwood M, Danielewska-Nikiel B, Borzelleca JF, Flamm GW, Williams GM, Lines TC. A critical review of the data related to the safety of quercetin and lack of evidence of in vivo toxicity, including lack of genotoxic/carcinogenic properties. Food and chemical toxicology 2007; 45(11): 2179-205.

42. Mattes RD, Bormann L. Effects of (-)-hydroxycitric acid on appetitive variables. Physiol Behav. 2000; 71(1): 87-94.
43. Sakariah KK, Jena BS, Jayaprakasha GK, Singh RP. Chemistry and biochemistry of (-)-Hydroxycitric acid from Garcinia. J of Agril Food Chem. 2002; 50(1): 10-22.

44. Deore AB, Vinayak D, Sapakal, Nilofer S, Naikwade. Antioxidant and hepatoprotective activity of garcinia indica linn fruit rind. International Journal of Comprehensive Pharmacy 2011; 6(8): 1-5.

45. Khatib NA, Pawase Kiran, Patil PA. Evaluation of anti-inflammatory activity of Garcinia Indica fruit rind extracts in wistar rats. International Journal of Research in Ayurveda and Pharmacy 2010; 1(2): 449-54. 